\title{
Active and Full-Handling Control of the Spatial MRI Phase Distribution with Optimal Control Theory
}

\author{
Pauline M. Lefebvre ${ }^{\mathrm{a}, 1, *}$, Eric Van Reeth ${ }^{\mathrm{a}, 1}$, Hélène Ratiney ${ }^{\mathrm{a}}$, Olivier Beuf ${ }^{\mathrm{a}}$, \\ Elisabeth Brusseau ${ }^{\mathrm{a}}$, Simon A. Lambert ${ }^{\mathrm{a}}$, Steffen J. Glaser ${ }^{\mathrm{b}}$, Dominique \\ Sugny c,d, Denis Grenier ${ }^{\mathrm{a}}$, Kevin Tse Ve Koon ${ }^{\mathrm{a}}$ \\ ${ }^{a}$ Univ.Lyon, INSALyon, Université Claude Bernard Lyon 1, UJM-Saint Etienne, CNRS, \\ Inserm, CREATIS UMR 5220, U1206, 3 rue Victor Grignard, F-69616, LYON, France \\ ${ }^{b}$ Department of Chemistry, Technische Universität München, Lichtenbergstraße 4, \\ D-85748 Garching, Germany \\ ${ }^{c} I C B$, UMR 6303 CNRS-Université de Bourgogne, 9 avenue Alain Savary, F-21078, \\ France \\ ${ }^{d}$ Institute for Advanced Study, Technische Universität München, Lichtenbergstraße 2a, \\ D-85748 Germany
}

\begin{abstract}
This paper lays the first stone on the use of Optimal Control theory to design Radio-Frequency (RF) pulses that actively control the spatial distribution of the MRI magnetization phase. The RF pulses are generated through the application of the Pontryagin Maximum Principle and optimized so that the resulting transverse magnetization reproduces various non-trivial and spatial phase patterns. Three different target-states are defined and the resulting optimal pulses are tested both numerically with the ODIN MRI

*Corresponding author at: Univ.Lyon, INSALyon, Université Claude Bernard Lyon 1, UJM-Saint Etienne, CNRS, Inserm, CREATIS UMR 5220, U1206, 3 rue Victor Grignard, F-69616, LYON, France

Email addresses: pauline.lefebvre@creatis.insa-lyon.fr (Pauline M. Lefebvre), eric.van-reeth@creatis.insa-lyon.fr (Eric Van Reeth), helene.ratiney@creatis.insa-lyon.fr (Hélène Ratiney), olivier.beuf@creatis.insa-lyon.fr (Olivier Beuf), elisabeth.brusseau@creatis.insa-lyon.fr (Elisabeth Brusseau), simon. lambert@creatis.insa-lyon.fr (Simon A. Lambert), steffen.glaser@tum.de (Steffen J. Glaser), dominique.sugny@u-bourgogne.fr (Dominique Sugny), denis.grenier@creatis.insa-lyon.fr (Denis Grenier), kevin.tsevekoon@creatis.insa-lyon.fr (Kevin Tse Ve Koon)

${ }^{1}$ These authors have contributed equally.
\end{abstract}


simulator and experimentally with an Agar gel phantom on a $4.7 \mathrm{~T}$ smallanimal MR scanner. Phase images obtained in simulations and experiments are both consistent with the defined phase patterns. A practical application of phase control with OC pulses is also presented, with the generation of RF pulses adapted for a Magnetic Resonance Elastography experiment. This study demonstrates the possibility to use optimal control RF pulses to encode information in the magnetization phase and thus opens the scope for accurate and active RF-based phase control in all MR fields using phase images.

Keywords: Optimal Control Theory, MRI phase, RF Pulses design, Phase control, MR elastography.

\section{Introduction}

Magnetic Resonance Imaging (MRI) techniques using the phase instead or in addition to the magnitude of the MR signal are increasingly developing. Phase images provide information on biological tissues that cannot be derived from magnitude images alone, as for example information on flow velocity [1] (phase-contrast cine MR imaging), magnetic susceptibility [2] (Susceptibility Weighted Imaging), viscoelastic properties [3] (Magnetic Resonance Elastography, MRE) or temperature [4] (MRI thermometry). Other phasebased sequences, as Diffusion Tensor MRI (DTI) [5], use spin phase shift to modify the magnitude signal intensity and thus extract information from signal attenuation. In these phase-based MRI techniques, the phase-encoding process is mainly handled with gradients, which often present physical limitations such as maximum amplitude or switching rate. For example, strong gradients are typically used in DTI sequences, and induce eddy currents [5]. This can cause geometrical distortion and therefore lead to significant errors in the estimation of diffusion parameters. In MRE, gradients have to be synchronized with the motion induced in the tissue and gradients commutations are physically limited at high frequencies: standard clinical systems can hardly go above $1 \mathrm{kHz}$.

The objective of this paper is to present a novel approach, exclusively based on RF pulses designed with Optimal Control (OC) theory in combination with a constant gradient, to encode the phase information. Magnetization phase is actively controlled, spatially and temporally, during the application of the RF pulse. The main benefit of this approach lies in the fact that the phase information is encoded during the excitation process: the signal 
can be immediately acquired with extremely short TE values. Classical approaches actually use actually post-excitation gradients for phase-encoding, and thus suffer from $T_{2}$ or $T_{2}^{\star}$ signal decay before acquisition.

Phase control with RF pulses has been investigated in some studies on selective pulse design. Linear-phase Shinar Le Roux pulses [6] can generate a linear phase pattern in the slice gradient direction. Carlson [7]-[8] used inverse scattering theory to design selective excitation pulses and control the phase distribution. His work aimed at finding a method to design pulses that give a flat phase distribution and that remove non-linear phase contributions but no general solution was presented to control the phase in a more general and non-linear case.

On the other hand, the use of OC for RF pulse design in MRI has already been performed in different contexts [9]: we can mention studies on robust (regarding field inhomogeneities) excitation and refocusing [10]-[13], contrast optimization [14]-[17] or parallel transmit [18]-[20]. The possibility to control the phase with OC has been mentioned and investigated in NMR studies [21]-[22] and has been applied to create delayed echo sequences in MRI [23]. However, to our knowledge the use of OC pulses to create a controlled macroscopic spatial phase distribution for imaging applications has not been explored. As a proof-of-concept, we design pulses achieving different non-trivial and spatial phase patterns, represented as different target-states in the transverse plane of the Bloch sphere (section III). These RF pulses are, in a second step, integrated into classical MRI sequences: simulations with the ODIN MRI simulator [24] (section IV) and experiments on a small-animal MRI scanner (section V) with Agar phantoms are performed.

A practical application of phase control with OC pulse is also presented in this article, with MRE technique (section VI): wave propagation information is directly encoded in the phase image with a RF pulse and a constant gradient, without post-excitation Motion-Encoding Gradients (MEG). A method for motion detection without MEG, has already been proposed in a previous paper [25]. This method is principally based on a hardware development (with a RF field gradient obtained from a ladder-shape coil) and can detect a mechanical vibration (and its direction, frequency and amplitude) but does not give a $2 \mathrm{D}$ phase image showing wave propagation. Our approach yields similar results to a classical MRE acquisition, using a software implementation (design of optimal RF pulse) and constant gradient (as opposed to switching gradients as it is the case in classical MRE). 


\section{Method: Optimal Control Theory}

\subsection{Optimal control theory framework in $M R I$}

Optimal control theory, with the application of the Pontryagin Maximum Principle (PMP) [26], enables the computation of the optimal trajectory of a dynamic system - with regards to a given optimality criterion - and its associated control.

In MRI application, the dynamic system corresponds to the macroscopic magnetization $\vec{M}=\left(M_{x}, M_{y}, M_{z}\right)$ of spin ischromats (defined in the Bloch sphere), whose evolution is governed by the Bloch equation:

$$
\frac{d}{d t}\left(\begin{array}{l}
M_{x} \\
M_{y} \\
M_{z}
\end{array}\right)=\left(\begin{array}{ccc}
-\frac{1}{T_{2}} & \triangle_{B_{0}} & -\omega_{y} \\
-\triangle_{B_{0}} & -\frac{1}{T_{2}} & \omega_{x} \\
\omega_{y} & -\omega_{x} & -\frac{1}{T_{1}}
\end{array}\right)\left(\begin{array}{l}
M_{x} \\
M_{y} \\
M_{z}
\end{array}\right)+\left(\begin{array}{c}
0 \\
0 \\
\frac{M_{0}}{T_{1}}
\end{array}\right)
$$

with $\triangle_{B_{0}}$ being the resonance offset, $T_{1}$ and $T_{2}$ the longitudinal and transverse relaxation times, $\left(\omega_{x}, \omega_{y}\right)$ the $x$ and $y$ components of the RF pulse (corresponding to the control field of this optimal control problem) that will bring the macroscopic magnetization to the user-defined target-state.

\subsection{Pontryagin Maximum Principle}

The OC problem is solved here with the application of the Pontryagin Maximum Principle [26]. The PMP is formulated from the definition of a pseudo-Hamiltonian:

$$
H=\vec{P} \cdot \dot{\vec{M}}
$$

where $\vec{P}$ is the costate vector. It can be interpreted in this optimization problem as a Lagrange multiplier associated to $\vec{M}$.

The PMP states that the optimal control $\omega^{\text {opt }}$ must minimize the Hamiltonian during the control time. Then, the optimal trajectories $\left(\vec{M}^{\text {opt }}\right.$ and $\vec{P}^{o p t}$ ) satisfy the following Hamiltonian equations:

$$
\dot{\vec{M}}^{o p t}=\frac{\partial H}{\partial \vec{P}^{o p t}} \quad \text { and } \quad \dot{\vec{P}}^{o p t}=-\frac{\partial H}{\partial \vec{M}^{o p t}}
$$

Moreover, the optimal trajectories must satisfy the following boundaries conditions:

$$
\vec{M}^{\text {opt }}\left(t_{0}\right)=\left(\begin{array}{c}
0 \\
0 \\
M_{0}
\end{array}\right) \quad \text { and } \quad \vec{P}^{o p t}\left(t_{f}\right)=\frac{\partial C}{\partial \vec{M}^{o p t}\left(t_{f}\right)}
$$


with $C$ being the cost function (defined by the user to achieve the desired target-state), $t_{0}$ (respectively $t_{f}$ ) the time at the beginning (respectively the end) of the control field.

\subsection{GRAPE algorithm}

This optimal control problem is solved with the GRAPE (Gradient Ascent Pulse Engineering) algorithm [27], which is a gradient ascent algorithm introduced for Nuclear Magnetic Resonance pulse design. It was implemented for this study on Matlab (The MathWorks, R2014b).

\subsubsection{Steps of the algorithm}

This part presents the different steps of the GRAPE algorithm as initially proposed. Starting from an initial control field (defined by the user), this algorithm optimizes the cost function iteratively by adjusting the control field at each iteration, while respecting the PMP constraints. The different steps are:

1. Choice of the initial control field $\omega^{0}$,

2. Forward propagation of $\vec{M}$, from $t_{0}$ to $t=t_{f}$, starting from $\vec{M}_{0}$ (defined from initial boundary condition (4)),

3. Backward propagation of $\vec{P}$, from $t=t_{f}$ to $t_{0}$, starting from $\vec{P}_{t_{f}}$ (defined from final boundary condition (4) and $\vec{M}_{t_{f}}$ ),

4. Update of the control field: computation of the following control field $\omega^{(k+1)}$ from control field $\omega^{(k)}$ with:

$$
\omega^{(k+1)}=\omega^{(k)}-\alpha \frac{\partial C}{\partial \omega}
$$

with $\alpha>0$ the convergence step,

5. Repetition of steps 2-4 until the convergence criterion is reached.

Gradient term $\frac{\partial C}{\partial \omega}$, in GRAPE algorithm, can be expressed as a function of the backward propagation of the costate vector and forward propagation of the magnetization vector and depends therefore only on two full time evolutions.

\subsubsection{Algorithm implementation details}

Gradient $\frac{\partial C}{\partial \omega}$ needs to be calculated with an accurate approximation in order to ensure fast and correct convergence. We use here complex differentiation [28]-[29] to estimate this cost function derivative. Let $f$ be a real 
function defined on real space $\left(f: \Re^{n} \longmapsto \Re\right)$, a small imaginary increment $i \varepsilon$ is added to $x$ and the Taylor series of $f(x)$ writes:

$$
f(x+i \varepsilon)=f(x)+i \varepsilon f^{\prime}(x)-\frac{\varepsilon^{2}}{2} f^{(2)}(x)+\ldots
$$

Assuming that $\varepsilon$ is small enough $(\varepsilon \ll 1), f^{\prime}(x)$ can be expressed as:

$$
f^{\prime}(x) \approx \frac{\operatorname{Im}(f(x+i \varepsilon))}{\varepsilon}
$$

The use of Equation 7 as a derivative approximation significantly improves the estimation accuracy compared to finite differences, which is affected by subtractive cancellation when small values of $\varepsilon$ are used. In the following sections, $\varepsilon$ is set to $10^{-10}$.

In the initial version of GRAPE algorithm, update of the control field (step 4) is performed iteratively with a constant convergence step $\alpha$. In this study, in order to improve the convergence speed and accuracy (compared to the simple gradient ascent optimisation method used in Equation 5), a second order approximation scheme was performed, with the implementation of a IBFGS quasi-Newton algorithm [30].

\section{RF pulses generation with OC for phase control}

\subsection{Definition of cost function}

Optimal control framework requires the formulation of a cost function, that will be minimized during the optimization process.

The purpose of this paper is to bring the magnetization of spins in a defined target-state in the transverse plane $\left(M_{X}, M_{Y}\right)$ of Bloch sphere. Therefore, we define the cost function to be minimized as:

$$
C\left(\omega_{x}, \omega_{y}\right)=\sum_{i}\left\|\vec{M}^{i}-\vec{M}^{i} T S\right\|^{2}
$$

This cost function tends towards zero as the distance between the magnetization of the $i^{\text {th }}$ spin $\vec{M}^{i}$ and its target-state $\vec{M}_{T S}^{i}$ decreases. In these defined target-states, magnitude of the magnetization is sought to be constant and as high as possible for all spins, whatever their positions on the transverse plane of Bloch sphere. 


\subsection{Definition of target-states and phase patterns}

For each spin, the target-state (i.e. state at the end of the optimal RF pulse) will depend on its position in readout direction. Spins positions are discriminated through the application of a gradient, which we shall call the phase sensitizing gradient, in the readout direction during the optimal $\mathrm{RF}$ pulse. Thus, the pulse will lead spins in a certain state in the transverse plane, depending on their perceived magnetic field (parameter $\triangle_{B_{0}}$ in Equation 1, which changes with the application of a gradient), and thus resonance offset $f_{i}$.

The frequency resonance offset range $\Delta f=\left[f_{i_{m i n}}, f_{i_{\text {max }}}\right]$ (proportional to the magnetic field offset) that should be controlled for a field of view $F O V_{x}$ in the readout direction (let us denote $x$ this direction) with the phase sensitizing gradient $G_{O C}$ applied during the OC pulse, is:

$$
\Delta f=\frac{\gamma}{2 \pi} \cdot G_{O C} \cdot F O V_{x}
$$

with $\frac{\gamma}{2 \pi}$ being the gyromagnetic ratio of proton (equal to $42.58 \mathrm{MHz} / \mathrm{T}$ ).

This resonance offset range, set to $10 \mathrm{kHz}$ for all calculated pulses, will be divided in three or four intervals. Each resonance offset interval will be assigned a target position in the transverse plane.

Three different non-trivial phase patterns in the transverse plane $\left(M_{X}, M_{Y}\right)$ of Bloch sphere were defined in this study, corresponding to different phase images patterns. One of these patterns consists in having three different phase evolutions along the readout direction: a linear phase increase, a constant level and a linear decrease. The two other patterns present four different areas, with different constant values of phase. They are represented in Figure 1 , with their corresponding profile along the readout direction.

More precisely, the first one (A) divides the readout direction in four areas, with respectively a magnetization phase equal to $0, \frac{\pi}{2}, \pi$ and $\frac{3 \pi}{2}$. The second one (B) defines four states in the transverse plane, in which phases are equal to $0, \pi, 0$ and $\pi$. Finally, the third target-state $(\mathrm{C})$ defines three areas along the readout direction: the phase of the first area increases linearly from 0 to $\pi$, the phase of the second one is constant and equal to $\pi$, and the phase of the last one decreases linearly from $\pi$ to 0 .

\subsection{RF pulses generation for phase control}

\subsubsection{Temporal resolution of the RF pulses}

As the optimal control problem gives here a numerical solution of the optimal RF pulses, they should be discretized in $\mathrm{N}$ time steps of duration 

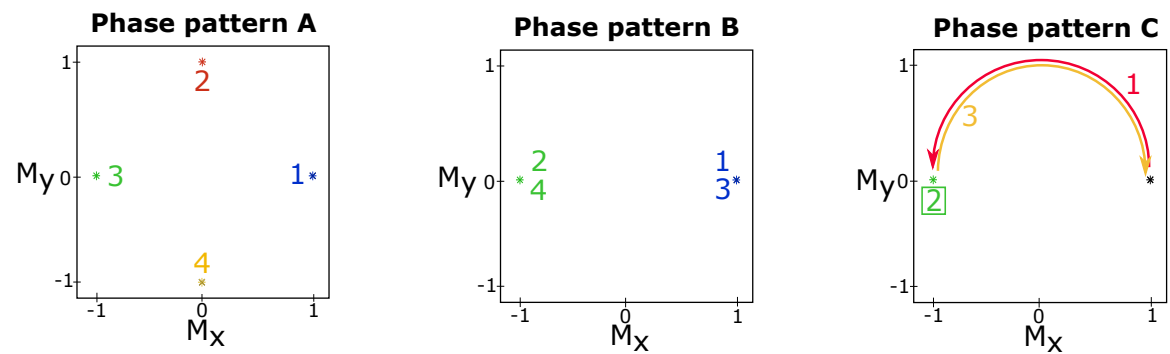

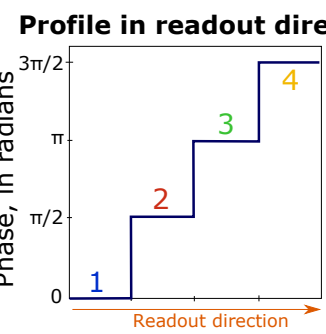

(a) Phase pattern $\mathrm{A}$

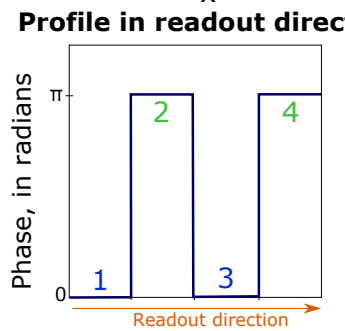

(b) Phase pattern B

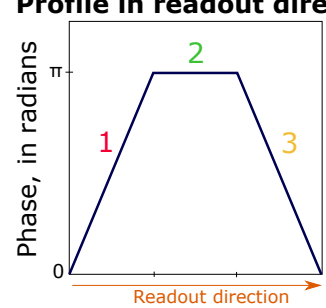

(c) Phase pattern $\mathrm{C}$

Figure 1: Three phase patterns defined for the phase control experiment with optimal control theory. Top row represents the spin target positions in the transverse plane $\left(M_{X}, M_{Y}\right)$ of the Bloch sphere. Bottom row displays the profile of spin phases along the readout direction.

$\Delta t$ during the time interval $\left[t_{0}, t_{f}\right]$. In this work, the following values were chosen: $N=300, t_{f}=6 \mathrm{~ms}$ and consequently, $\Delta t=20 \mu \mathrm{s}$.

\subsubsection{Relaxation times}

RF pulses were generated with $T_{1}$ and $T_{2}$ values (that appear in Bloch equation, and so in the forward and backward propagation in GRAPE algorithm) corresponding to the one of the Agar gel phantom (Agar concentration $1.5 \%$ in weight) used in the MRI experiments, i.e. $T_{1}=1500 \mathrm{~ms}$ and $T_{2}=130$ ms (see below).

\subsubsection{Optimal RF pulses}

Figures 2, 3 and 4 present the three computed RF pulses $\mathrm{A}, \mathrm{B}$ and $\mathrm{C}$ (magnitude and phase as a function of time). These figures also show the simulated transverse magnetization trajectories of spins during the application of computed pulses, for different spins belonging to the various defined offset frequency intervals (and so final spin positions). For clarity purpose, the spins trajectories of pulse $\mathrm{C}$ are divided in three plots, corresponding to the three intervals (linear increase, constant level and linear decrease of 

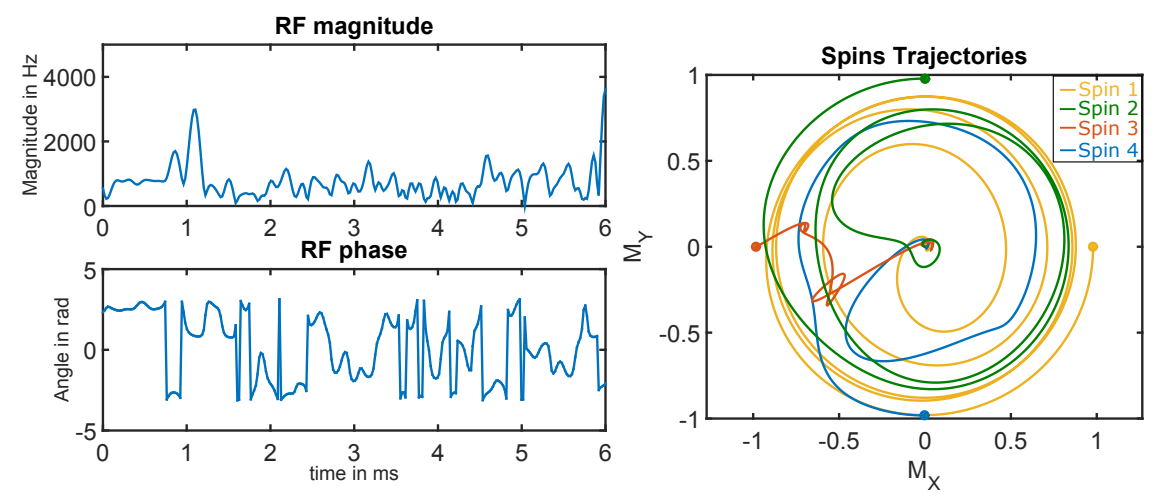

Figure 2: Magnitude and phase of the optimal RF pulse associated to target-state A as a function of time. Projections in the transverse plane of the trajectories of selected spins belonging to the different frequency intervals are displayed on the right: Spin 1 (resp. 2, 3 and 4) corresponds to a resonance offset belonging to the first (resp second, third and fourth) area of the pattern displayed on figure 1a. Final states are marked with a dot.
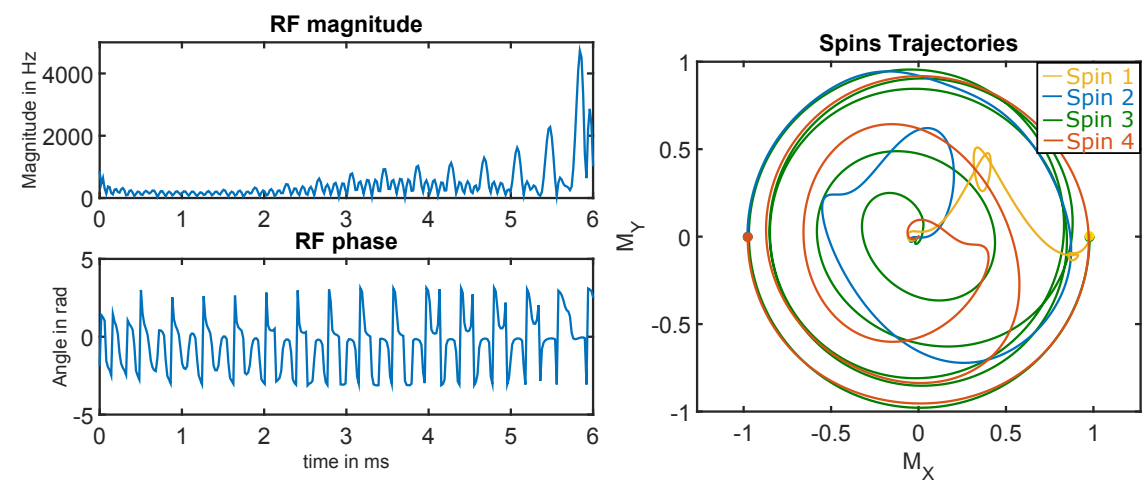

Figure 3: Magnitude and phase of the optimal RF pulse associated to target-state B as a function of time. Projections in the transverse plane of the trajectories of selected spins belonging to the different frequency intervals are displayed on the right: Spin 1 (resp. 2, 3 and 4) corresponds to a resonance offset belonging to the first (resp second, third and fourth) area of the pattern displayed on figure 1b. Final states are marked with a dot.

phase). Final states are marked with a dot and are consistent with the target-states defined on Figure 1.

For the RF pulse corresponding to phase pattern $\mathrm{C}$, the resulting phase for different resonance offsets (blue asterisks) is presented on figure 5 and compared to the profile of the target phase (dark line). This figure highlights the correct convergence of the algorithm: the resulting magnetization phase 

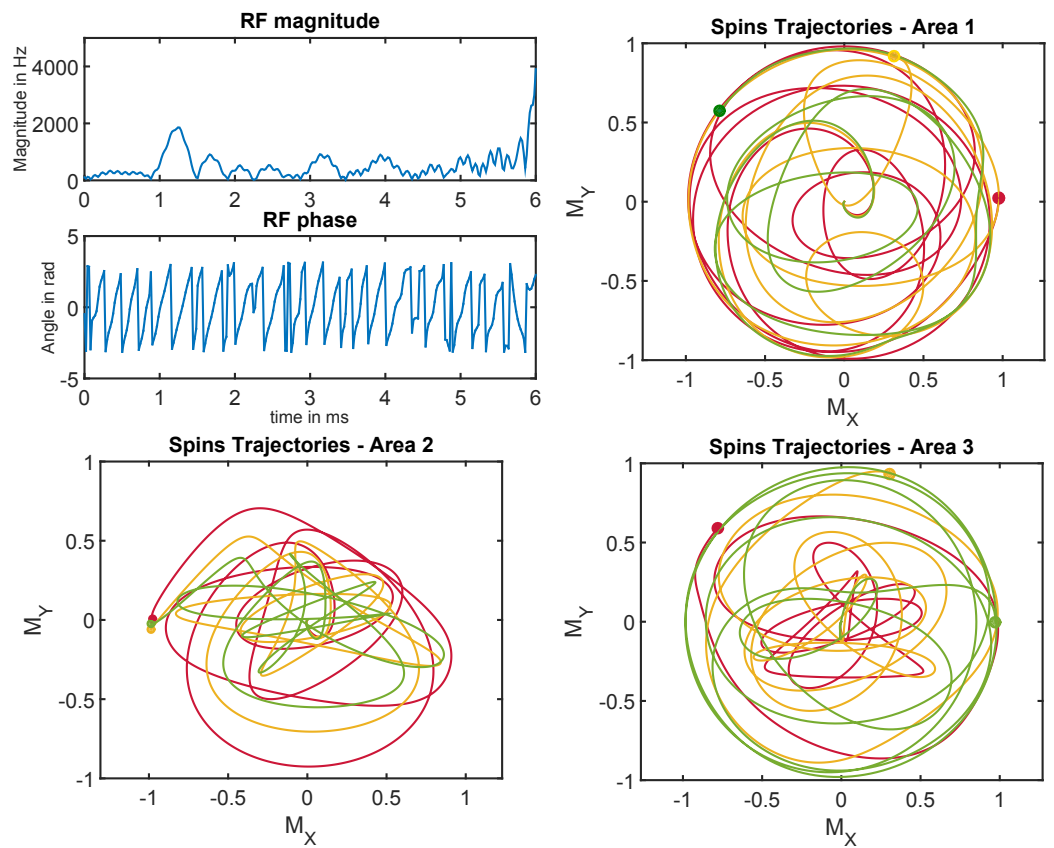

Figure 4: Magnitude and phase of the optimal RF pulse associated to target-state C as a function of time. Projections of the spins trajectories in the transverse plane of the Bloch sphere are displayed on different figures for each target-state area (see on Figure 1c): three different resonance offsets were taken in each of the three areas of the pattern. Final states are marked with a dot.

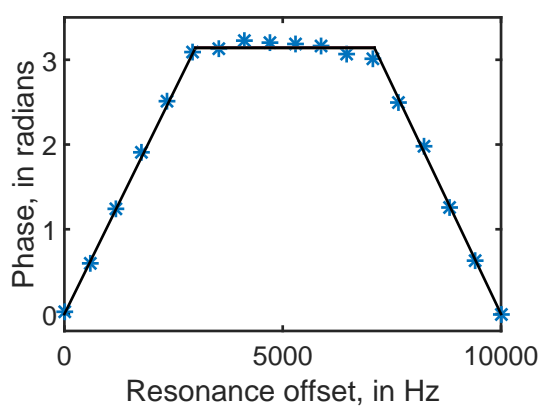

Figure 5: Phase profile as a function of resonance offset (as a reminder, frequency resonance offset range is set to $10 \mathrm{kHz}$ in all pulses), obtained from pulse $\mathrm{C}$ (blue asterisks), and compared to the profile of the target phase pattern of pulse $\mathrm{C}$ (dark line).

associated with pulse $\mathrm{C}$ is very close to the profile of the target phase patterns. 


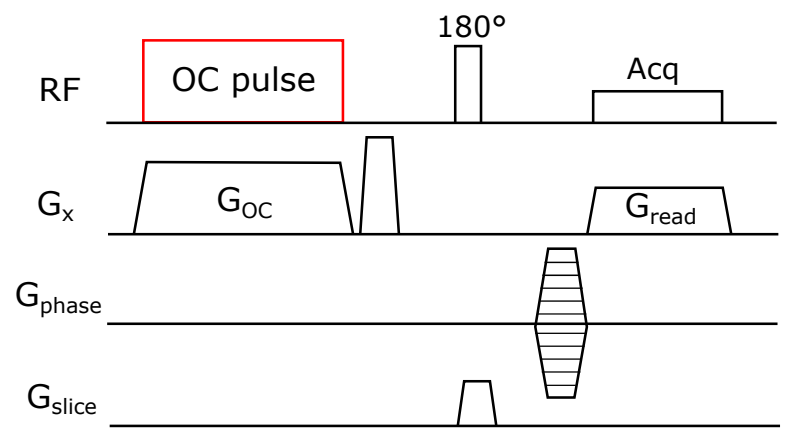

Figure 6: ODIN spin-echo sequence adapted for the optimal control pulse experiment.

\section{MRI simulations of phase control with ODIN}

\subsection{Simulation conditions}

Simulations were performed using the MRI simulator ODIN [24]. ODIN is a $\mathrm{C}++$ software framework designed to develop and simulate MR sequences. Once a virtual phantom and a MRI sequence have been specified, it propagates the Bloch equations in the defined virtual phantom throughout the whole sequence.

\subsubsection{Generation of virtual phantom}

Virtual phantom was generated while specifying constant $T_{1}, T_{2}$ and proton density maps. The $T_{1}$ and $T_{2}$ values remain constant on the whole maps (corresponding to an assumption of homogeneous phantom) and are equal to the relaxation times of the Agar phantom used in MRI experiments. Dimensions of the virtual phantom were set to $20 \times 20 \times 3 \mathrm{~mm}$.

\subsubsection{Sequence parameters}

A spin-echo sequence, adapted for the OC pulse experiment, was implemented in ODIN. The phase sensitizing gradient $G_{O C}$ was applied simultaneously with the $\mathrm{OC}$ pulse, to discriminate spins positions along the readout direction. The slice selective gradient, applied usually during the excitation pulse, was removed, but the refocusing pulse was kept selective. A chronogram of the sequence is presented on Figure 6.

The echo-time was set to the minimum value possible in order to allow maximum signal, and was fixed to $\mathrm{TE}=6.6 \mathrm{~ms}$. The repetition-time was sufficiently long to ensure full longitudinal magnetization relaxation, and was 
fixed to $\mathrm{TR}=5 \mathrm{~s}$. The phase sensitizing gradient $\left(G_{O C}\right)$ was fixed to 11.7 $\mathrm{mT} / \mathrm{m}$, according to Equation 9 with frequency range $\Delta f=10 \mathrm{kHz}$ and $F O V_{x}=20 \mathrm{~mm}$. Bandwidth of the acquisition was equal to $25 \mathrm{kHz}$. Matrix size was $64 \times 64$ pixels.

\subsection{Results}

Magnitude and phase images resulting from ODIN simulations are presented on Figure 7, for the three optimal RF pulses A, B, C (plotted on Figures 2, 3 and 4). Moreover, mean phase of each column on the phase images has been calculated and the resulting profiles plotted.

For these three pulses, phase and magnitude images are consistent with the expected results. Notice that the slight phase decrease in area 2 of pulse $\mathrm{C}$ is consistent with the profile obtained from pulse $\mathrm{C}$, as shown on figure 5. Standard deviations of the magnitude images were calculated over the whole phantom, excluding phase transitions areas, and are presented in Table 1: signal magnitude presents very small standard deviations for the three pulses and is therefore nearly constant, as expected. For each area of phase images (excepted areas 1 and 3 of pulse C), a Region of Interest (ROI) was defined by taking all pixels within the considered area, and mean and standard deviations were extracted. Results are presented in Table 1. Phase values in the different areas correspond to the expected one.

For the linear phase variations of pulse $\mathrm{C}$ (area 1 and 3 ), mean of each phase image column has been calculated and the obtained profile has been linearly fitted to a linear curve. The coefficient of determination, $R^{2}$, has been extracted, with the slope value normalized to the frequency offset control range (10 kHz divided in three intervals). The high values obtained for $R^{2}$ (values close to 1) indicate that phase variations are linear in these two areas and the slope in both areas are close to the expected ones (expected slopes: $\pm \frac{\pi}{10 / 3} \mathrm{rad} / \mathrm{kHz}= \pm 0.942 \mathrm{rad} / \mathrm{kHz}$ ).

The phase images of pulses A and B present sharp transitions between the different areas. This demonstrates that optimal RF pulses accurately control the phase, with good frequency selectivity. It is also interesting to notice that in sharp phase transition areas, a decrease of magnitude signal is observed, due to the signal cancellation that occurs when summing out-of-phase spins. 


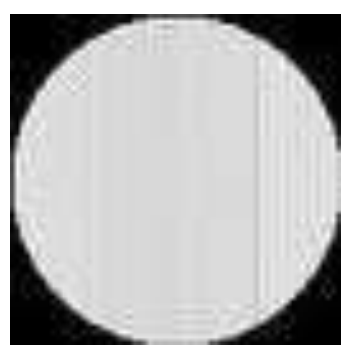

(a) Magnitude - Pulse A

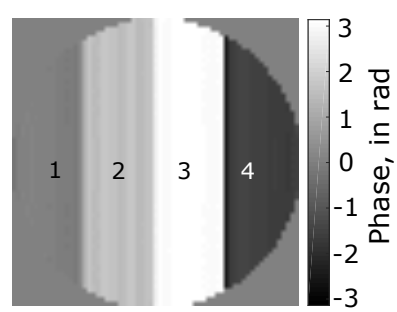

(d) Phase - Pulse A

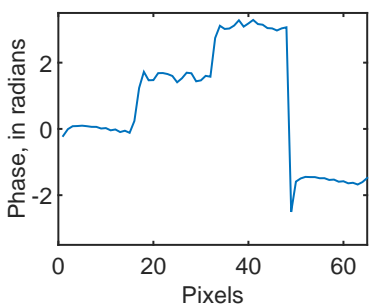

(g) Phase profile - Pulse A

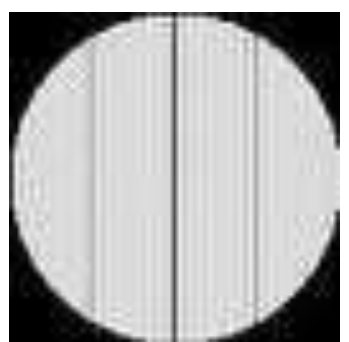

(b) Magnitude - Pulse B

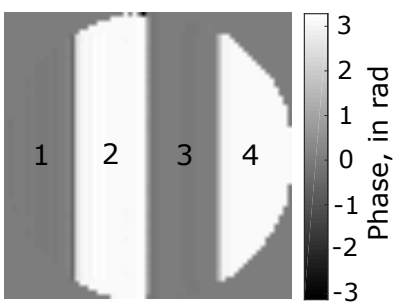

(e) Phase - Pulse B

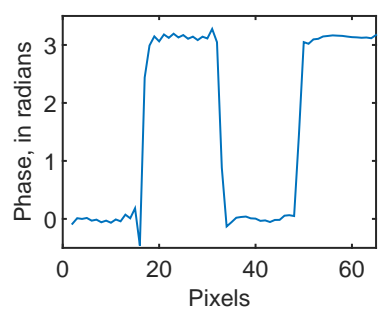

(h) Phase profile - Pulse B

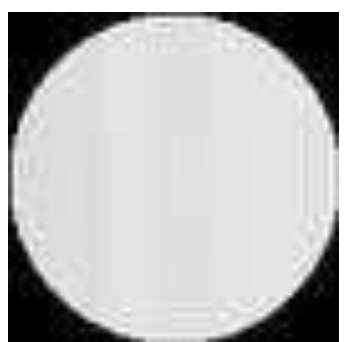

(c) Magnitude - Pulse C

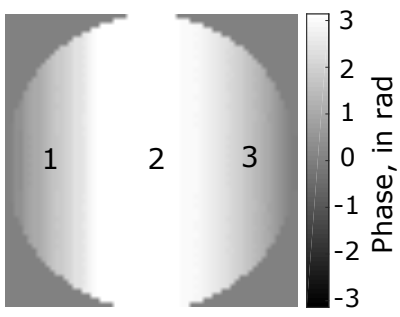

(f) Phase - Pulse C

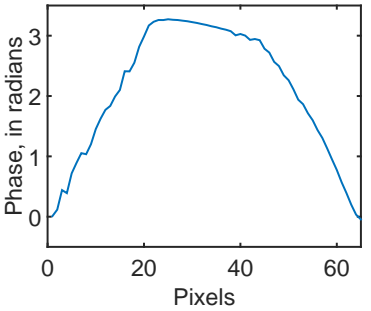

(i) Phase profile - Pulse C

Figure 7: Magnitude and phase (in radians) images obtained from ODIN MRI simulations, with the three RF pulses $\mathrm{A}, \mathrm{B}$ and $\mathrm{C}$ and corresponding mean phase profiles on bottom row.

\section{MRI experiments on phase control}

\subsection{Experimental conditions}

Experiments were carried out on a homogeneous Agar phantom (Agar concentration $1.5 \%$ in weight). $T_{1}$ and $T_{2}$ values were measured using an exponential fit of the water peak acquired with a localized PRESS spectroscopy sequence, for different TE and TR. They were found to be $T_{1}=1500 \mathrm{~ms}$ and 
Table 1: Standard deviations of magnitude images and average phase angles in radian and corresponding standard deviations obtained from the three different pulses A, B and C (figures 7 and 8).

\begin{tabular}{|c|c|c|c|c|}
\hline & \multirow{3}{*}{ ODIN } & \multirow{3}{*}{ MRI } \\
\hline & & & & \\
\hline \multirow{6}{*}{ Pulse A } & Area & Expected phase & & \\
\hline & 1 & 0 & $0.04 \pm 0.06$ & $-0.19 \pm 0.08$ \\
\hline & 2 & $\pi / 2$ & $1.57 \pm 0.03$ & $1.41 \pm 0.18$ \\
\hline & 3 & $\pi$ & $3.12 \pm 0.05$ & $3.18 \pm 0.15$ \\
\hline & 4 & $3 \pi / 2=-\pi / 2$ & $-1.55 \pm 0.01$ & $-1.78 \pm 0.17$ \\
\hline & \multicolumn{2}{|c|}{ Standard deviation of magnitude } & $3.0 \%$ & $6.6 \%$ \\
\hline \multirow{6}{*}{ Pulse B } & Area & Expected phase & & \\
\hline & 1 & 0 & $-0.01 \pm 0.14$ & $-0.16 \pm 0.17$ \\
\hline & 2 & $\pi$ & $3.13 \pm 0.06$ & $2.99 \pm 0.07$ \\
\hline & 3 & 0 & $-0.01 \pm 0.18$ & $-0.20 \pm 0.08$ \\
\hline & 4 & $\pi$ & $3.12 \pm 0.13$ & $3.02 \pm 0.07$ \\
\hline & \multicolumn{2}{|c|}{ Standard deviation of magnitude } & $4.0 \%$ & $8.6 \%$ \\
\hline \multirow{7}{*}{ Pulse C } & Area & Expected phase and linearity & & \\
\hline & 1 & $R^{2}$ value & 0.9865 & 0.9951 \\
\hline & 1 & Slope (exp. $0.942 \mathrm{rad} / \mathrm{kHz}$ ) & 0.950 & 0.969 \\
\hline & 2 & $\pi$ & $3.12 \pm 0.11$ & $3.02 \pm 0.09$ \\
\hline & 3 & $R^{2}$ value & 0.9988 & 0.9961 \\
\hline & 3 & Slope (exp. $-0.942 \mathrm{rad} / \mathrm{kHz})$ & -0.893 & -0.888 \\
\hline & \multicolumn{2}{|c|}{ Standard deviation of magnitude } & $3.1 \%$ & $7.3 \%$ \\
\hline
\end{tabular}

$T_{2}=130 \mathrm{~ms}$. The phantom was cylindrically-shaped, with a diameter equal to $27 \mathrm{~mm}$ and a height of $50 \mathrm{~mm}$.

MRI measurements were run on a Bruker 4.7 T small-animal MRI system, with a cylindrical inner-diameter $30 \mathrm{~mm}$ bird-cage mouse coil. A spin-echo sequence, similar to the one used for ODIN simulations (Figure 6), was used with $64 \times 64$ matrix and FOV $=3.25 \times 3.25 \mathrm{~cm}^{2} . G_{O C}$ was set to $8 \mathrm{mT} / \mathrm{m}$, according to Equation 9, with $\Delta f=10 \mathrm{kHz}$ for the RF pulses. Optimal RF pulses used in the following three MRI experiments were exactly the same as the ones used for the ODIN simulations. The echo-time was set to $9.8 \mathrm{~ms}$ and the repetition-time to $5 \mathrm{~s}$. 


\subsection{Slice selectivity}

The optimal RF pulses are applied without slice selective gradients. Therefore the obtained signal comes from the overall sample. In order to get rid of refocused artefactual signal coming from extremities of the phantom, spatial saturation bands have been added in the slice direction and, similarly to the Odin simulation, the refocusing pulse has been kept selective.

\subsection{Results}

Figure 8 presents the resulting magnitude and phase images obtained from MRI measurements, for the three optimal pulses A, B and C. Mean phase of each column on the phase images has been calculated and the resulting profiles are also presented on Figure 8.

Here again, magnitude and phase images are consistent with expected results. Resulting magnitude images are nearly homogeneous in the whole phantom, as shown on Table 1. They present however some inhomogeneities close to the coil, corresponding to the magnetic field inhomogeneities coming from the copper rungs of the volume coil. Phase values, presented in Table 1, also correspond well to the defined target-states. Note that a slight difference of less than $0.2 \mathrm{rad} . \mathrm{s}^{-1}$ can be observed between expected values and obtained phase patterns.

Finally, resulting phase images present the same sharp profiles than the ones obtained in simulation, showing that the RF pulses accurately control the phase in real conditions.

\section{An example of application: Magnetic Resonance Elastography}

Results presented in previous parts validate the possibility to master the magnetization phase with RF pulses, in a simple experiment with a static resonance offset applied (through a gradient) during the pulse. A practical application of this phase control is now presented with the generation of RF pulses adapted for Magnetic Resonance Elastography. This is a more complex framework as, in this application, the resonance offset $\triangle B_{0}$ is timedependant and thus changes during the application of the RF pulse.

\subsection{Introduction on Magnetic Resonance Elastography}

Magnetic Resonance Elastography is a MR non-invasive method enabling characterization of viscoelastic properties of tissues [3]. Shear waves are induced in the tissue of interest using an external driver. Tissue motion is 


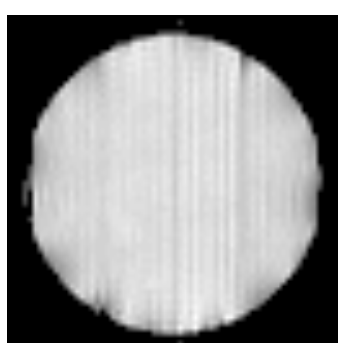

(a) Magnitude - Pulse A

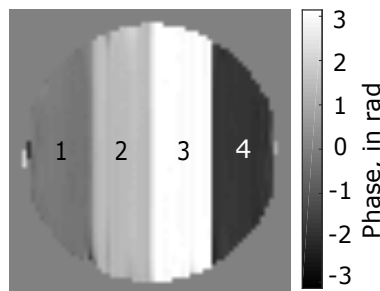

(d) Phase - Pulse A

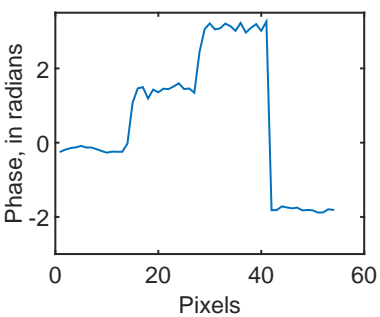

(g) Profile phase - Pulse A

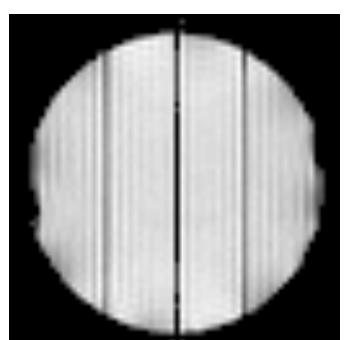

(b) Magnitude - Pulse B

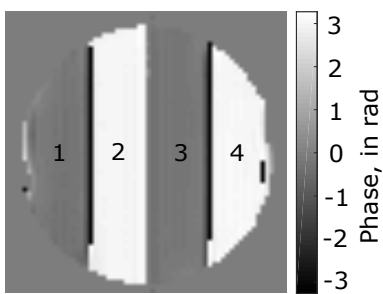

(e) Phase - Pulse B

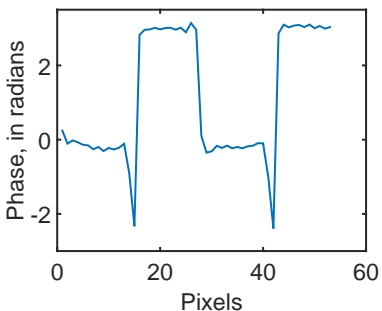

(h) Profile phase - Pulse B

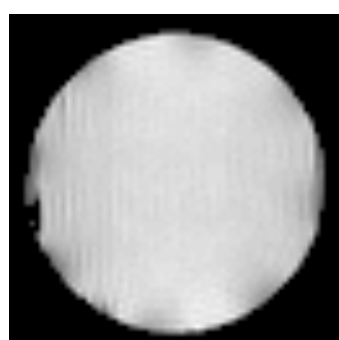

(c) Magnitude - Pulse C

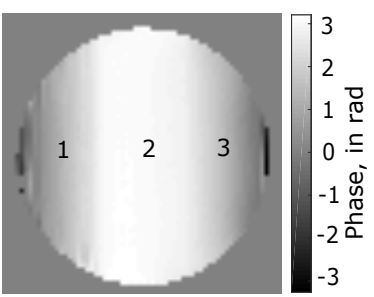

(f) Phase - Pulse C

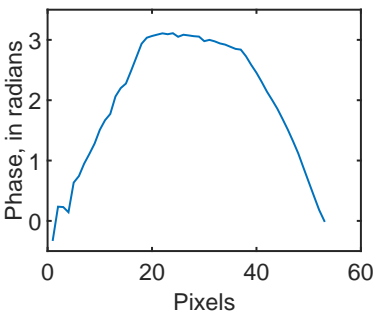

(i) Profile phase - Pulse C

Figure 8: Magnitude and phase (in radians) images obtained from MRI measurements, with the three optimal RF pulses $\mathrm{A}, \mathrm{B}$ and $\mathrm{C}$ and corresponding mean phase profiles on bottom row.

then encoded in phase images with a motion-sensitive MR imaging sequence and these images are finally processed to map and quantify the viscoelastic properties of the tissue.

In MRE sequences, a periodic gradient, called Motion-Encoding Gradient (MEG) is applied synchronously with the external harmonic excitation, typically at the same frequency and along the direction of the cyclic motion of spins. The cyclic spin motion in combination with the MEG causes a measurable phase-shift in the NMR signal, proportional to the scalar product 


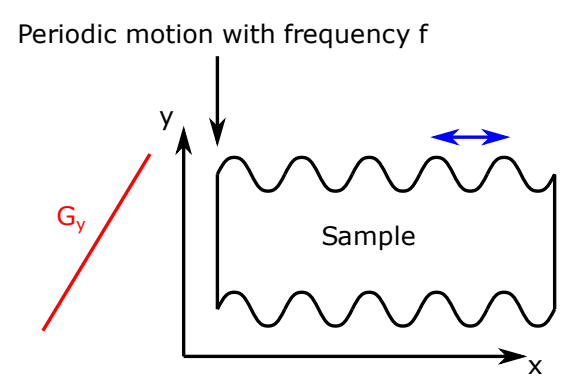

(a) Wave propagation

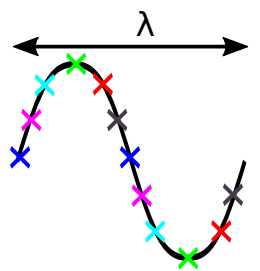

(b) Discretization

Figure 9: a) Wave propagation pattern at a given fixed time. The wave propagates in the $x$-direction and spin motion is in the $y$-direction. A gradient, $G_{y}$, is applied in the $y$-direction. This wave oscillates at frequency $f_{e}$, corresponding to the wavelength $\lambda . \mathrm{b}$ ) Discretization of one wavelength into 12 phase offsets.

of the displacement vector and the MEG vector, and to the duration of the MEG. Acquired phase images correspond to a snapshot of the mechanical wave propagation within the tissue.

\subsection{Adaptation of Bloch equations for MRE}

In presence of a gradient in the motion direction (the MEG), motion induced by shear waves creates a variation in the perceived magnetic field by spins. Figure $9 \mathrm{a}$ presents the wave propagation and coordinate system used here for the definition of the optimal control problem.

Variation of the time-dependent perceived magnetic field can be expressed as the product of the oscillating motion and the MEG:

$$
\Delta B_{0}(x, y, t)=G_{y}(t) \times A \times \sin \left(-2 \pi f_{e} t+2 \pi \frac{x}{\lambda}\right)
$$

with $y$ the cyclic spin motion direction, $G_{y}$ being the amplitude of the gradient (here $y$ ), $x$ the direction of wave propagation, $A$ the amplitude of periodic motion, $f_{e}$ the motion frequency, and $\lambda$ the wavelength.

During the optimal RF pulse, a constant gradient $G_{y}(t)=G_{y}^{0}$ will be applied in the motion direction. The dynamic resulting resonance offset $\triangle B_{0}(t)$ is integrated in the Bloch equations (Equation 1) during the optimization process.

\subsection{Definition of target-state and cost function}

$\mathrm{W}$ propose to design a RF pulse that encodes wave propagation in the phase image. For that purpose, we define a cost function that: i) linearly 


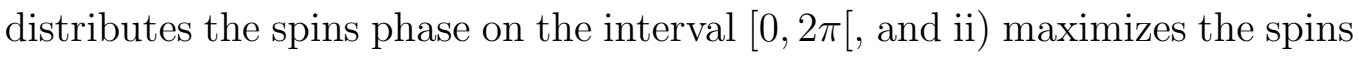
transverse magnetization. The successive target states thus lie on the unit circle of the normalized transverse magnetization plane. Let $N$ be the number of considered spins equally spaced along a wavelength of the propagation wave (Figure 9b), and the spin index $s \in[0, N-1]$, the cost function writes:

$$
C\left(\omega_{x}, \omega_{y}\right)=\sum_{s=0}^{N-1}\left\|\vec{M}_{s}^{\perp}\left(t_{f}\right)-\vec{T}_{s}\right\|^{2}
$$

with $\vec{T}_{s}$ the target-state vector (in the transverse plane), defined as:

$$
\vec{T}_{s}=\left(\begin{array}{c}
\cos \left(s \times \frac{2 \pi}{N}\right) \\
\sin \left(s \times \frac{2 \pi}{N}\right)
\end{array}\right)
$$

Depending on their positions along the wavelength, spins will be assigned a different magnetization phase target-state.

\subsection{RF pulse generation}

\subsubsection{RF pulse parameters}

The RF pulse was optimized for a motion amplitude $\mathrm{A}=9 \mu \mathrm{m}$, and for an excitation frequency $f_{e}=600 \mathrm{~Hz}$. Constant gradient in the spin motion direction was set to $8 \mathrm{mT} / \mathrm{m}$. Pulse was optimized by discretizing the wavelength in $N=10$ spins (cf. Equations 11 and 12). Its duration was fixed to $40 \mathrm{~ms}$. It should be noted that this duration is just an example and can be optimized for a shorter duration.

\subsubsection{Pulse bandwidth}

Since we are interested in producing $2 \mathrm{D}$ images and since a constant gradient is applied to encode the spins motion, it seems natural to use the latter as our slice selective gradient. Therefore, the optimal pulse must ensure a consistent spins distribution throughout the whole bandwidth corresponding to the desired slice thickness. In practice, this is done by discretizing a frequency interval, on which the pulse is optimized, and by increasing iteratively the frequency interval (and thus increasing the total number of spins to be controlled), in order to control a larger slice thickness. The slice thickness is directly determined by the considered frequency range (bandwidth), and the strength of the gradient applied in slice direction (that is also the motion direction). 

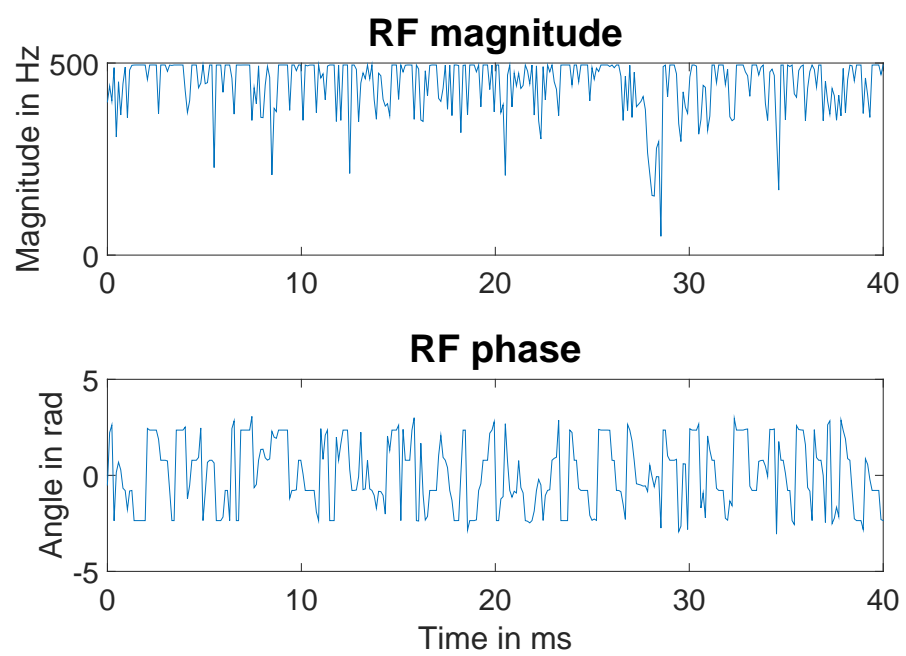

Figure 10: Magnitude and phase of the optimal RF pulse associated to the elastography problem, as a function of time.

\subsubsection{MRI coil physical constraints}

$\mathrm{RF}$ pulses calculated through OC theory must respect practical constraints of the coils and the RF amplifiers used in experiments, in particular their peak amplitude. Thus, during optimization process, the maximum RF amplitude was constrained to $500 \mathrm{~Hz}$, corresponding to an attenuation of 9.8 $\mathrm{dB}$ with the $70 \mathrm{~mm}$ volume coil used in MRI experiments.

. Figure 10 presents the generated RF pulse (magnitude and phase).

\subsection{Simulation with $O D I N$}

A dynamic numerical phantom, with dimensions $4 \times 4 \mathrm{~cm}^{2}$, was generated with pre-defined $T_{1}$ and $T_{2}$ maps (same as for MRI experiments, see below). The effect of the motion in the virtual phantom was directly encoded as a $B_{0}$ field shift (in ppm) [31], given by:

$$
\Delta B_{0}(x, t)=\frac{10^{6}}{B_{0}}\left(A G \times \sin \left(2 \pi f_{e} t\right) \times \sin \left(2 \pi f_{e} t-2 \pi \frac{x}{\lambda}\right) \times e^{-\alpha x}\right)
$$

with $B_{0}$ the static magnetic field, and $\lambda$ the wavelength of the propagating wave set to $10 \mathrm{~mm}$. Exponential term $\alpha$ represents the attenuation of the wave during its propagation, due to viscosity effects, and was fixed to 50 


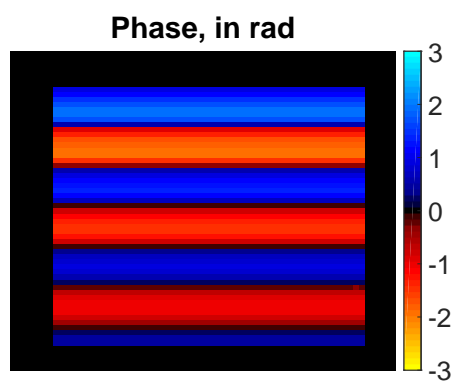

(a) Phase image

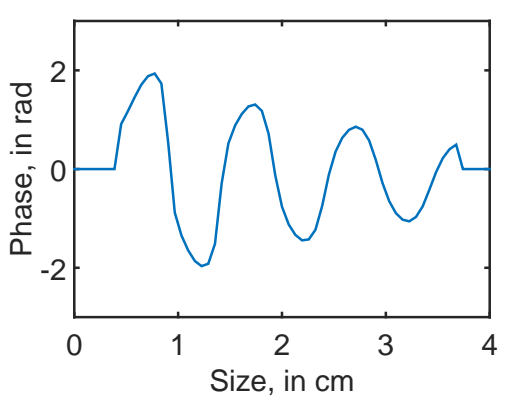

(b) Phase profile

Figure 11: Phase image (in radians) obtained from the optimal RF pulse associated to the elastography problem, with the ODIN MRI simulation, and its corresponding profile.

$\mathrm{m}^{-1}$. A gradient-echo was used: the echo-time was set to $4 \mathrm{~ms}$ and the repetition-time to $1500 \mathrm{~ms}$.

Obtained phase image, with the optimal RF pulse (plotted on Figure 10), is presented on Figure 11 with its profile along the motion direction. Wave propagation pattern is clearly visible on this figure, with an attenuation of phase during its propagation from top to bottom, and the wavelength corresponds to the value attributed to the phantom during phantom generation (Equation 13).

\subsection{MRE experiment}

MRE experiment was performed on the Bruker 4.7 T MRI system, with a $70 \mathrm{~mm}$ volume coil. Shear waves were induced in the phantom using a noninvasive custom-made device maintaining the medium between two plates [32], the upper one being fixed while the lower one being activated by a piezoelectric actuator (CEDRAT Technologies). Acquisition was performed for an excitation frequency equal to $600 \mathrm{~Hz}$, corresponding to the one used for the pulse generation.

A standard plastisol phantom was prepared (Plastileurre Standard, Bricoleurre, France), its response to excitation frequency at $600 \mathrm{~Hz}$ was well known [32]. $T_{1}$ and $T_{2}$ values were found to be 300 and $25 \mathrm{~ms}$ respectively.

The MR sequence was a gradient-echo sequence, with an echo-time equal to $5.2 \mathrm{~ms}$, a repetition-time set to $1500 \mathrm{~ms}$, a FOV $=4 \times 4 \mathrm{~cm}^{2}, 32 \times 32$ matrix, and $\mathrm{NA}=8$. 


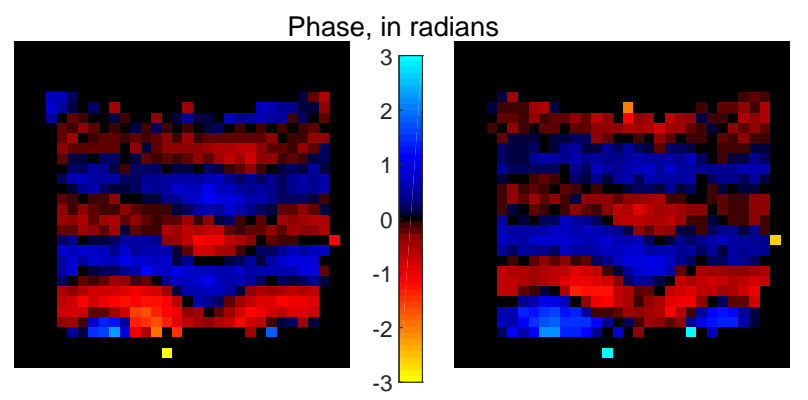

Figure 12: Phase images (in radians) obtained from MRE experiments, at two different time steps of wave propagation ( $\pi / 2$ phase shift between these two images).

Phase-opposite wave images were acquired and then subtracted, as performed in usual MRE experiments to remove static phase contributions. Resulting phase images, at two different time steps of the wave propagation, are displayed in Figure 12. Notice that no filtering was applied to these phase images. Despite the presence of noise, wave pattern is clearly visible on this figure and consistent with the expected wavelength at $600 \mathrm{~Hz}(9 \mathrm{~mm})$ for plastisol phantom [32]. The non-plane wave aspect (compared to the ones obtained with ODIN) is due to inhomogeneities of the mechanical excitation surface and similar wave patterns were obtained with classical MRE acquisition.

\section{Discussion}

Our goal in this paper was to explore the use of RF pulses, designed with optimal control theory, to actively control the MR phase signal.

First, resulting phase images in sections 4 and 5 present a very good agreement with the desired phase patterns, in both simulations and experiments on phantom. These initial experiments validate the ability of optimal RF pulses to control the magnetization phase based on the spins oscillation frequency. Note that in this study, the phase pattern was arbitrarily created in the readout direction. The results can be extended to any other dimension with the same RF pulses.

Computation times required for the design of the RF pulses $\mathrm{A}, \mathrm{B}$ and $\mathrm{C}$ depend on the frequency range offset. Running the algorithm on a $4 \mathrm{x}$ 2.7 GHz machine using Matlab (The MathWorks, Inc., Natick, MA, USA, $\mathrm{R} 2014 \mathrm{~b})$ takes less than fifteen minutes for a frequency range of $10 \mathrm{kHz}$, with 
a discretization of $50 \mathrm{~Hz}$ (201 spins considered in the frequency range offset), as it has been chosen in this study.

The current implementation of the optimization algorithm requires a unique magnetization state as a boundary condition, which was chosen here as the thermal equilibrium. Thus, the acquisition strategy requires long TRs, as we have to wait for full longitudinal relaxation after each excitation, before performing the next RF pulse. However a turbo spin echo sequence could be used to accelerate the acquisition.

After these initial experiments, the use of optimal RF pulses in a more practical framework was investigated. MRE was chosen as a proof-of-concept, and results presented in section 6 show, for the first time, the possibility to to acquire MRE phase images with RF pulses, and a constant gradient.

A first advantage of using optimal RF pulses here is the very short echotime achievable. In standard MRE sequences, motion-encoding gradients typically last between 5 and $10 \mathrm{~ms}$ and lengthen therefore the echo-time. During this time, magnetization relaxation occurs and this echo-time increase can be critical for some tissues with very short $T_{2}$, as in the healthy mouse liver where $T_{2}$ value is equal to about $20 \mathrm{~ms}$, or at low frequency (where duration of the MEG is longer).

Moreover, using OC RF pulses relaxes the constraint on having a periodic gradient, and thus avoids fast gradients switching which is a hardware limitation.

Slice selectivity is controlled in this MRE case by the combination of a frequency offset range (on which the pulse has been optimized) and a gradient in the slice direction, that is also the motion direction. However, it is hard to predict the behaviour of the spins that lie outside the control range. This could explain the noise observed in the MRE phase images in Figure 12. Results presented here on MRE are preliminary but encouraging, as wave propagation is clearly visible on phase images. Future improvements will imply a better understanding of spin behaviour outside the control range, and the investigation of new cost functions definition.

\section{Conclusion}

This work validates the use of optimal control RF pulses to accurately and actively control the magnetization phase in MRI. As a proof-of-concept, RF pulses were computed to create non-trivial phase patterns. Both simulation and experiments on phantom present good match with the desired pattern. 
Optimal RF pulses were also designed in the context of MR elastography, to show their interest in a more practical framework. Preliminary results demonstrate, for the first time, that optimal RF pulses can be used to encode the dynamic wave propagation in the presence of a constant gradient.

RF-based phase encoding would present several advantages over standard techniques based on gradient switching (shorter TE, reduction of eddy currents, ability to overcome limitations due to gradient switching rates). This work can open new research projects on MRI phase-based sequences, with the use of RF pulses that directly encode physical and biochemical information in the MR phase image.

\section{Acknowledgment}

This work is supported by the ANR-DFG research program Explosys (Grant No. ANR-14-CE35-0013-01; GL203/9-1) and from the Technische Universität München Institute for Advanced Study, funded by the German Excellence Initiative and the E. U. Seventh Framework Programme under Grant No. 291763. This work was performed within the framework of the LABEX PRIMES (ANR-11-LABX-0063/ANR-11-IDEX-0007).

\section{References}

[1] N.J Pelc, R.J. Herfkens, A. Shimakawa, D.R. Enzmann, Phase contrast cine magnetic resonance imaging, Magn Reson Q. 1991 Oct;7(4):229-54.

[2] E.M. Haacke, Y. Xu, Y.-C. N. Cheng, J.R. Reichenbach, Susceptibility weighted imaging (SWI), Magn Reson Med. 2004 Aug;52(3):612-18. DOI:10.1002/mrm.20198.

[3] R. Muthupillai, D.J. Lomas, P.J. Rossman, J.F. Greenleaf, A. Manduca, R.L. Ehman, Magnetic resonance elastography by direct visualization of propagating acoustic strain waves, Science. 1995 Sept;269:1854-57. DOI:10.1126/science.7569924.

[4] J. De Poorter, C. De Wagter, Y. De Deen, C. Thomsen, F. Stahlberg, E. Achten, Noninvasive MRI Thermometry with the Proton Resonance Frequency (PRF) Method: In Vivo Results in Human Muscle, Magn Reson Med. 1995 Jan;33(1):74-81. DOI:10.1002/mrm.1910330111. 
[5] P.J. Basser, D.K. Jones, Diffusion-tensor MRI: theory, experimental design and data analysis - a technical review, NMR Biomed. 2002 Nov;15(7-8):456-67. DOI:10.1002/nbm.783.

[6] J. Pauly, P. Le Roux, D. Nishimura, A. Macovski, Parameter Relations for the Shinnar-Le Roux Selective Excitation Pulse Design Algorithm, IEEE Trans Med Im. 1991 March;10(1):53-65. DOI:10.1109/42.75611.

[7] J.W. Carlson, Exact solutions for Selective Excitation Pulses. II. Excitations Pulses with Phase Control, J Magn Res. 1992 March;97(1):65-78. DOI:10.1016/0022-2364(92)90237-2.

[8] J.W. Carlson, Nonlinear Phase Adjustment of Selective Excitation Pulses, J Mag Res. 2000 Dec;147(2):210-6. DOI:10.1006/jmre.2000.2201.

[9] S.J. Glaser, U. Boscain, T. Calarco, C.P. Koch, W. Köckenberger, R. Kosloff, I. Kuprov, B. Luy, S. Schirmer, T. Schulte-Herbrüggen, D. Sugny, F.K. Wilhelm, Training Schrödinger's cat: quantum optimal control - Strategic report on current status, visions and goals for research in Europe, Eur Phys J D. 2015 Dec;69:279. DOI:10.1140/epjd/e201560464-1.

[10] S. Conolly, D. Nishimura, A. Macovski, Optimal control solutions to the magnetic resonance selective excitation problem, IEEE Trans Med Im. 1986 June;5(2):106-115. DOI:10.1109/TMI.1986.4307754.

[11] T. E. Skinner, T. O. Reiss, B. Luy, N. Khaneja, S. J. Glaser, Application of optimal control theory to the design of broadband excitation pulses for high-resolution NMR, J Magn Res. 2003 Jul;163(1):8-15. DOI:10.1016/S1090-7807(03)00153-8.

[12] T. E. Skinner, N. I. Gershenzon, M. Nimbalkar, W. Bermel, B. Luy, S. J. Glaser, New strategies for designing robust universal rotation pulses: application to broadband refocusing at low power, J Magn Res. 2012 Mar;216:78-87. DOI:10.1016/j.jmr.2012.01.005.

[13] C. S. Aigner, C. Clason, A. Rund, R. Stollberger, Efficient high resolution RF pulse design applied to simultaneous multi-slice excitation, J Magn Res. 2016 Feb;263:33-44. DOI:10.1016/j.jmr.2015.11.013. 
[14] B. Bonnard, O. Cots, S. Glaser, M. Lapert, D. Sugny, Y. Zhang, Geometric optimal control of the contrast imaging problem in nuclear magnetic resonance, IEEE Trans Automatic Control. 2012 Aug;57(8):1957-69. DOI:10.1109/TAC.2012.2195859.

[15] M. Lapert, Y. Zhang, M. Janich, S. Glaser, D. Sugny, Exploring the physical limits of saturation contrast in magnetic resonance imaging, Sci Rep 2. 2012;589. DOI:10.1038/srep00589.

[16] B. Bonnard, M. Claeys, O. Cots, P. Martinon, Geometric and numerical methods in the contrast imaging problem in nuclear magnetic resonance, Acta Applicandae Mathematicae. 2014:135(1);5-45. DOI:10.1007/s10440-014-9947-3.

[17] E. Van Reeth, H. Ratiney, M. Tesch, S. J. Glaser, D. Sugny, Optimizing MRI contrast with B1 pulses using optimal control theory, IEEE 13th International Symposium on Biomedical Imaging, 2016 (Prague). DOI:10.1109/ISBI.2016.7493271.

[18] D. Xu, K. F. King, Y. Zhu, G. C. McKinnon, Z.-P. Liang, Designing multichannel, multidimensional, arbitrary flip angle RF pulses using an optimal control approach, Magn Res Med. 2008 Mar;59(3):547-60. DOI:10.1002/mrm.21485.

[19] A. Massire, M. A. Cloos, A. Vignaud, D. L. Bihan, A. Amadon, N. Boulant, Design of non-selective refocusing pulses with phasefree rotation axis by gradient ascent pulse engineering algorithm in parallel transmission at 7T, J Magn Res. 2013 May;230:76-83. DOI:10.1016/j.jmr.2013.01.005.

[20] A. Sbrizzi, H. Hoogduin, J. V. Hajnal, C. A. van den Berg, P. R. Luijten, S. J. Malik, Optimal control design of turbo spin-echo sequences with applications to parallel-transmit systems, Magn Res Med. 2017 Jan;77(1):361-73. DOI:10.1002/mrm.26084.

[21] K. Kobzar, B. Luy, N. Khaneja, S.J. Glaser, Pattern Pulses: Design of Arbitrary Excitation Profiles as a Function of Pulse Amplitude and Offset, J Magn Reson. 2005 Apr;173(2):229-35. DOI:10.1016/j.jmr.2004.12.005. 
[22] N. I. Gershenzon, T. E. Skinner, B. Brutscher, N. Khaneja, M. Nimbalkar, B. Luy, S.J. Glaser, Linear Phase Slope in Pulse Design: Application to Coherence Transfer, J Magn Reson. 2008 June;192(2):235-43. DOI:10.1016/j.jmr.2008.02.021.

[23] J. Assländer, S. J. Glaser, J. Hennig, Spin Echoes in the Regime of Weak Dephasing, Magn Res Med. 2016 Jan;75(1):150-60. DOI:10.1002/mrm.25579.

[24] T.H. Jochimsen, M. Von Mengershausen, ODIN-object-oriented development interface for NMR, J Magn Res. 2004 July;170(1):67-78. DOI:10.1016/j.jmr.2004.05.021.

[25] N. Baril, C.J. Lewa, J.D. de Certaines, P. Canioni, J.-M. Franconi, E. Thiaudière, MR Detection of Mechanical Vibrations Using a Radiofrequency Field Gradient, J Magn Res. 2002 Jan;154(4):22-7. DOI:10.1006/jmre.2001.2451.

[26] L.S. Pontryagin, B. Boltyanskii, R. Gamkrelidze, E. Mishchenko, The mathematical theory of optimal processes, Wiley-interscience, New York, 1962.

[27] N. Khaneja, T. Reiss, C. Kehlet, T. Schulte-Herbruggen, S. J. Glaser, Optimal control of coupled spin dynamics: design of NMR pulse sequences by gradient ascent algorithms, J Magn Res. 2005 Feb;172(2):296-305. DOI:10.1016/j.jmr.2004.11.004.

[28] M. Lapert, On GRAPE implementation, Private Note, April 2016.

[29] W. Squire and G. Trapp, Using complex variables to estimate derivatives of real functions, Siam Review. 1998;40(1):110-12. DOI:10.1137/S003614459631241X.

[30] P. de Fouquieres, S. Schirmer, S. Glaser, I. Kuprov, Second order gradient ascent pulse engineering, J Magn Res. 2011 Aug:212(2):412-17. DOI:10.1016/j.jmr.2011.07.023.

[31] P.M. Lefebvre, E. Van Reeth, E. Brusseau, D. Grenier, K. Tse Ve Koon, Magnetic Resonance Elastography simulation with an Object Oriented Development Interface for NMR, 33rd annual ESMRMB Congress. Vienna (Austria), 2016. DOI:10.1007/s10334-016-0568-x. 
[32] P.M. Lefebvre, K. Tse Ve Koon, E. Brusseau, S. Nicolle, J.F. Palierne, S.A. Lambert, D. Grenier, Comparison of Viscoelastic Property Characterization of Plastisol Phantoms with Magnetic Resonance Elastography and High-Frequency Rheometry, IEEE 38th Conference of the Engineering in Medicine and Biology Society, 2016 (Orlando):1216-19. DOI:10.1109/EMBC.2016.7590924. 